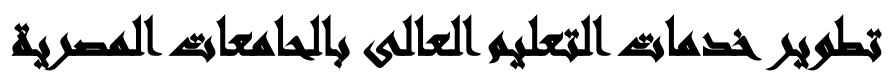

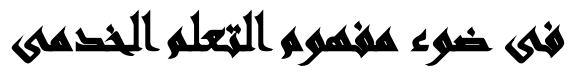

\section{$[1 \wedge]$}

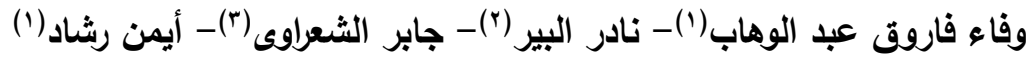

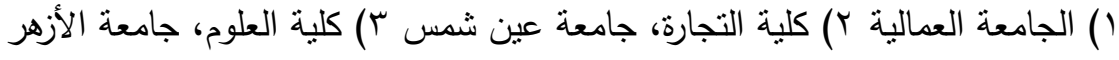

\section{المستخلتص}

على الرغم من أهمية التعليم في جميع أنحاء العالم العربي بشكل عام وعلى الرغم من الرنا

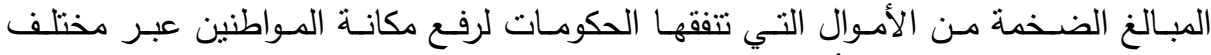

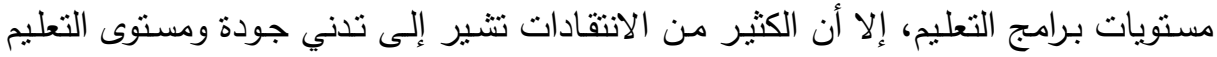

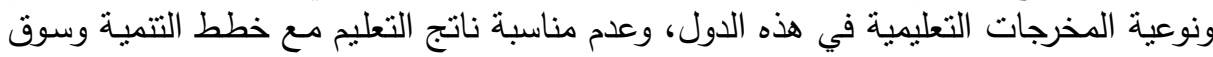

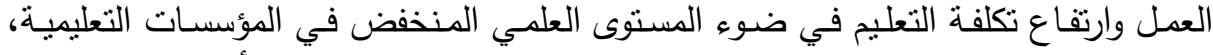

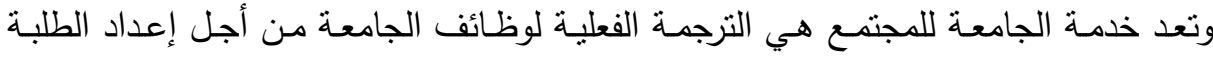

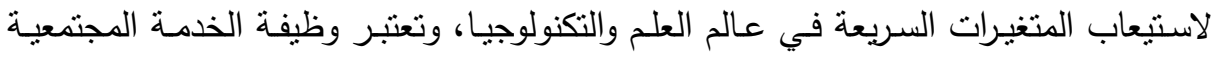

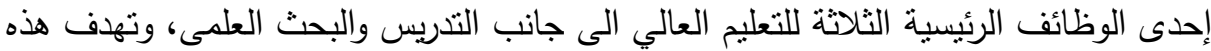

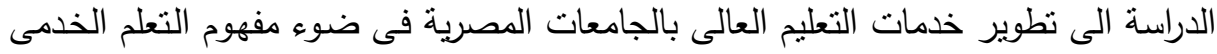

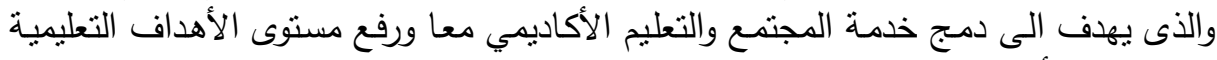

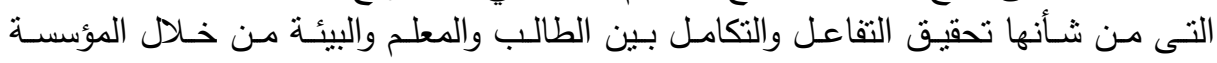

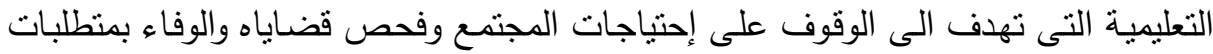

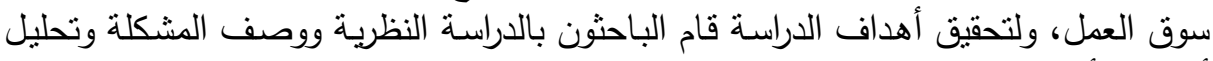

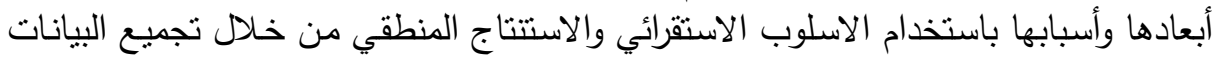

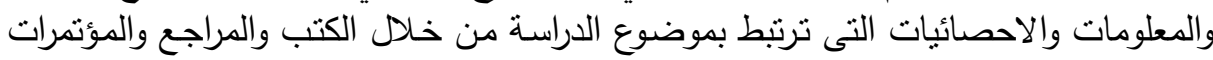

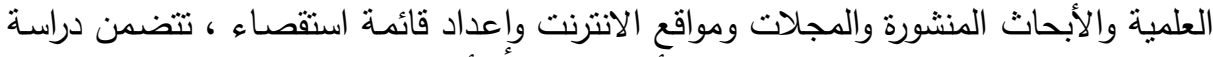

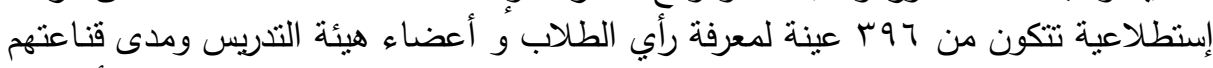

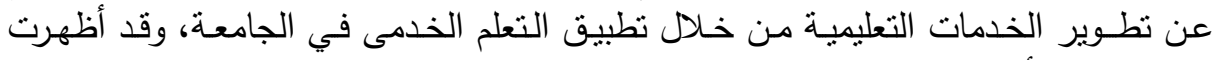

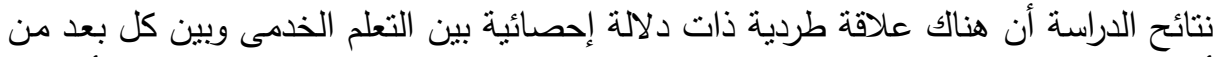

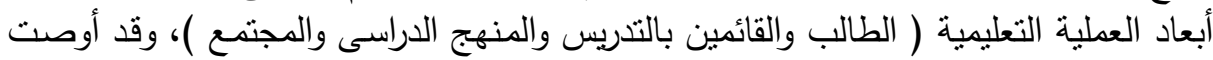

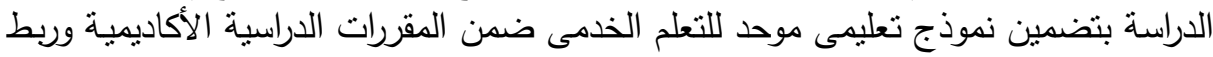

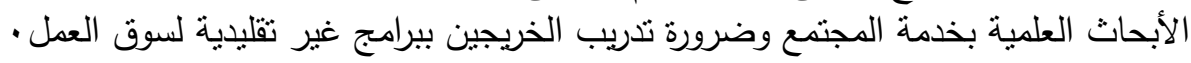

$$
\text { المجلد السادس والأربعون، الجزء الثالث، يونيو } 9 \text { ـ ب ب }
$$




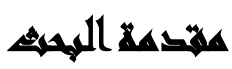

يعانى النظام التعليمى المصرى لكثير من المشاكل والتحديات والتى تمثل عائقا أمام العملية التعليمية وتطورها ولا يخفى علينا ان الطلاب تشتكى من أسلوب التدريس باستخدام

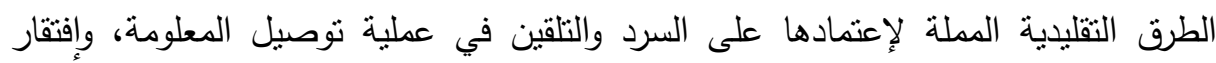

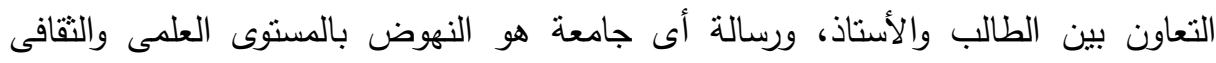

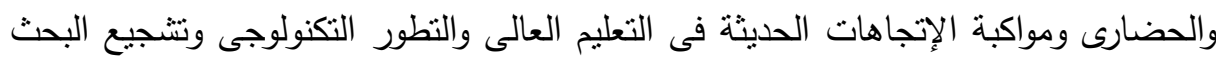
العلمى فى بناء الأجيال وتتمية المجتمع، ومن هنا ظهرت الحاجة الى نماذج تعليم جديدة تمد

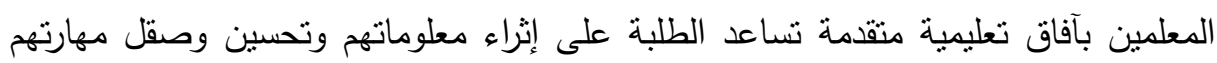
والمساهمة فى تيسير دخولهم الى مجال العمل بكل يسر والارتقاء بمستوى الأهداف التعليمية

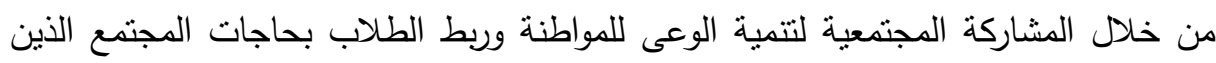

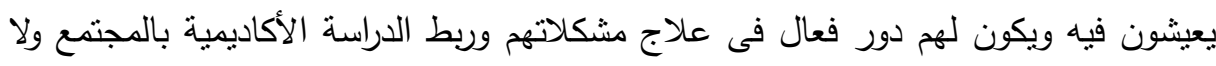

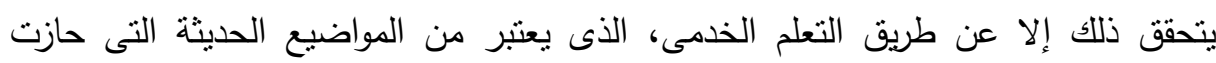

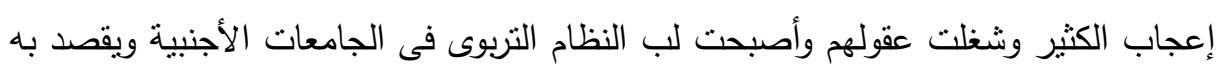
الإسهامات والأنشطة التى يقدمها الطلاب لتتمية المجتمع وحل بعض مشكلاته كأعضاء

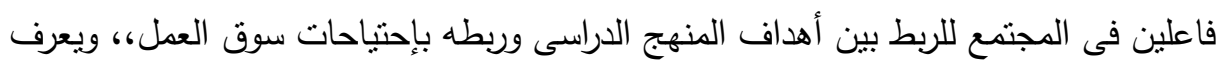
المركز الوطنى بأمريكا ( National Service Learning House ) التعلم الخدمى بأنه خبرات منظمة من التعلم تجمع بين تعلم خدمة المحتمع وأهداف التعليم الممنهحة، ويتم فيه مشاركة بين الطلاب والمجتمع بصورة مباشرة وإدراك العلاقة بين التعليم الأكاديمى والخدمة فئمة

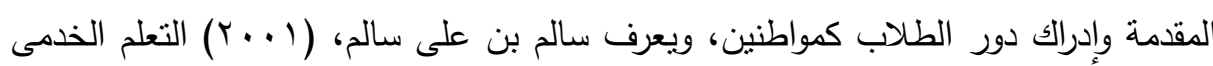

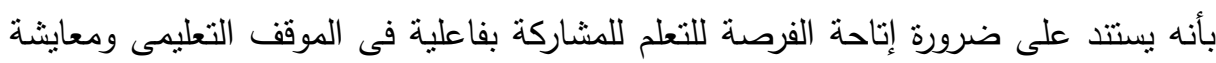

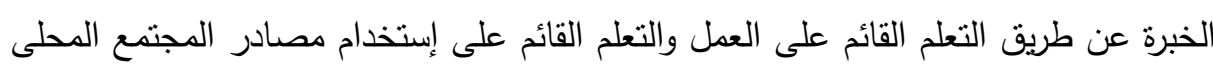

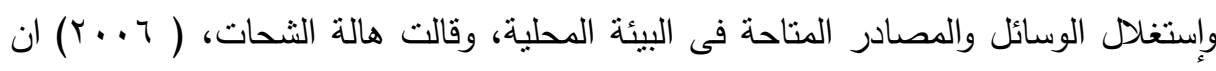

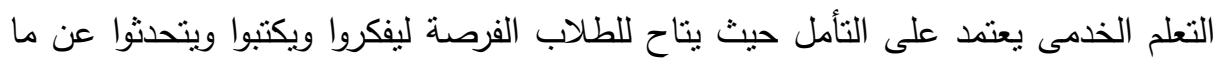

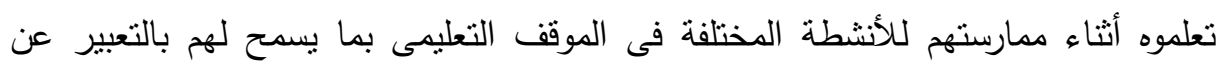

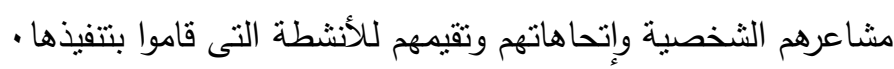




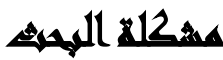

بالرغم أن بعض الدول قد خطت خطوات كبيرة لجعل الجامعة في خدمة المجتمع المحلى، كما فى اليابان حيث تقدم الكليات المتوسطة junior colleges حوالي ...0 كلية برامج تستغرق عامين في ميادين تتصل بتتمية المجتمع والعمل على خدمته، وهذه البرامج تتمنل في تعليم الأفراد حفظ الطعام، والتربية في رياض الأطفال والتصوير فقد جاء فين في في أحد التقارير الصادرة عن دور الجامعات اليابانية في المجتمع المحلى أن القدر المتحقق في لعاي الإسهام في أنشطة المجتمع بين كل من الجامعات العامة والخاصة محدود وضئيل للغاية وذللك على الرغم من ضخامة أبحاثها التقليدية باترشيا هـ - كروسون ( 1917 ) )

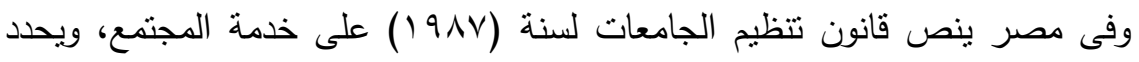
وظيفة الجامعة بأنها تختص بكل ما يتعلق بالتعليم الجامعي والبحث العلمي الذي ثقوم به

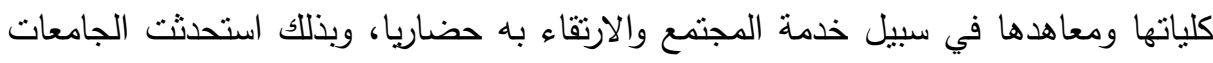

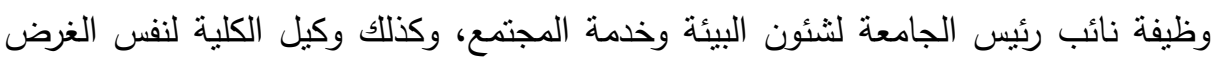

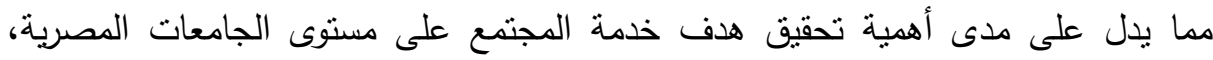

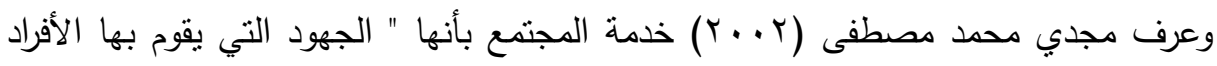

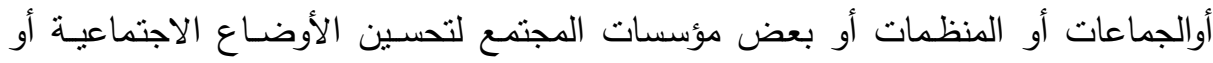

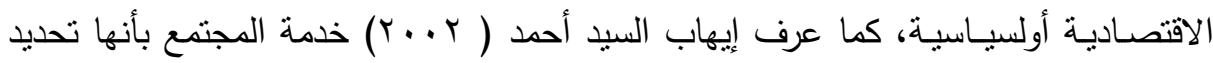

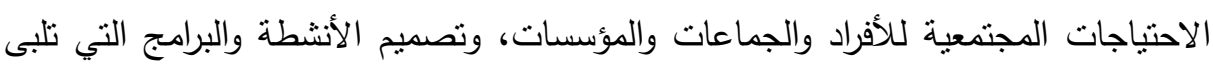
هذه الاحتباجات عن طريق الجامعة وكلياتها، ومراكزها البحثية المختلفة بغية إحداث تغيرات

$$
\text { تتموية وسلوكية مرغوب فيها. }
$$

كما أثنارت أيضا وحدة البحوث الاجتماعية والتربوية في عمادة البحث العلمي (.... ب) وعرفت بأنها " تلكك العملية التي يتم من خلالها تمكين أفراد المجتمع وجماعاته ومؤسساته وهيئاته من تحقيق أقصى استفادة ممكنة من الخدمات المختلفة التي تقدمها الجامعة بوسائل وأساليب منتوعة تتتاسب مع ظروف المستفيد وحاجاته الفعلية، كما تعرف المجالس القومية

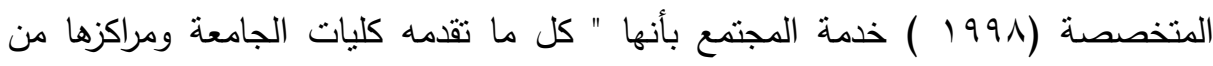

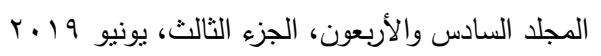


أنشطة وخدمات تتوجه بها إلى غير طلابها النظامين أو أعضاء هيئة التدريس بها، من أفراد

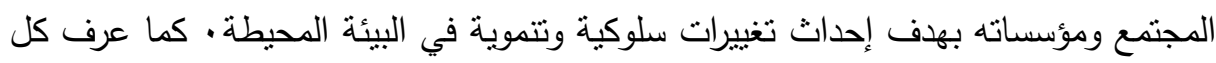

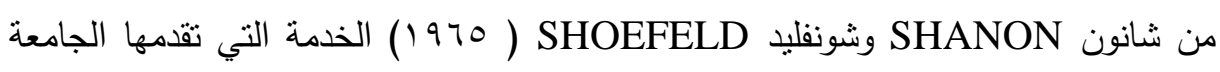
لمجتمعاتها على أنها " نشاط ونظام تعليمي موجه إلى الغير طلاب الجامعة، ويمكن عن طريقة نشر المعرفة خارج جدران الجامعة وذلك بغرض إحداث تغيرات سلوكية وتتموية في

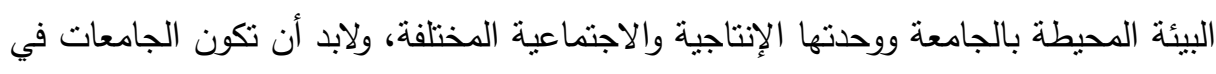

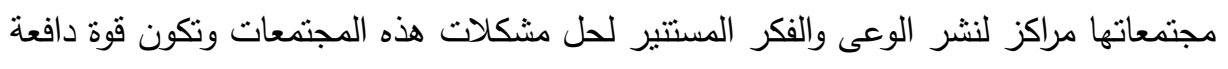

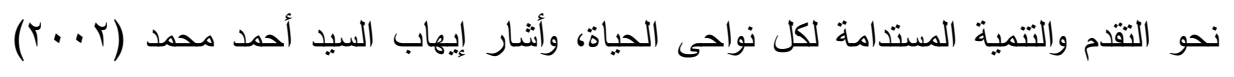
بتتوع مجالات خدمة المجمع وتعددها طبقا لظروف وإمكانيات كل جامعة على حدة وكذلك

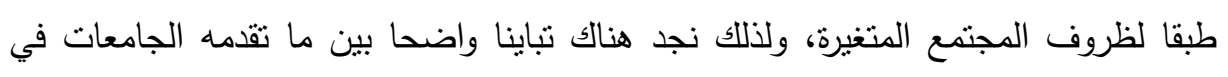
هذا المجال وأيا كانت تلك المجالات فإنها عبارة عن أنشطة وممارسات بهدف تحقيق والتئ التمية

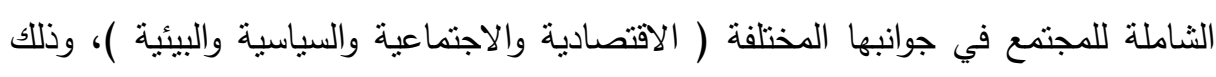
عن طريق استغلال كل القدرات الفعلية والمصادر المادية لمؤسسات التعليم العالي لتحسين

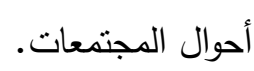

وتكمن مشكلة الدراسة فى إيجاد نموذج استرشادي يهدف الى دمج خدمة المجتمع والتعليم الأكاديمى عن طريق التعلم الخدمى بهدف تتمية معلومات واتجاهات الطلبة وإكسابهم

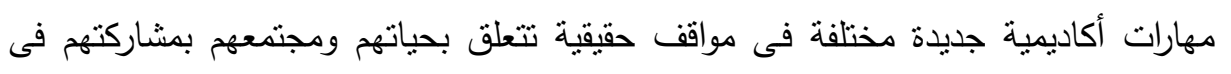
المجتمع بحيث تكون الششاركة مبنية على خبرات تعليمية منظمة ومدروسة لتحقيق احتياجات

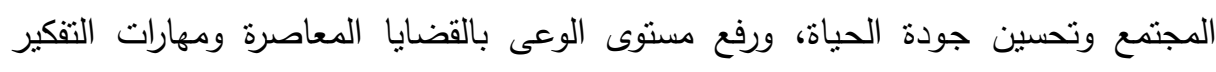
المستقبلى.

\section{أسئلة الهمهي}

1- ب - ما هو مفهوم التعلم الخدمى؟

r- ما أهمية نطوير خدمات العملية التعليمية عن طريق التعلم الخدمى؟ 


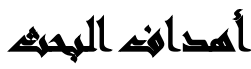

يهدف البحث الى تطوير خدمات التعليم العالى بالحامعات المصرية فى ضوء مفهوم التعلم الخدمى بما يحقق التفاعل والتكامل بين الطالب والمدرس والبيئة المحيطة -

\section{هزوضر الهمهي}

يوجد فرق ذو دلالة إحصائية عند مستوى معنوية $\alpha=$ ( (.,.)) بين التعلم الخدمى وتتمية مهارات الطلبة وقدراتهم على التواصل وإكسابهر مهارات أكاديمية حديدة تتعلق بمحتمعهم وبين التعلم الخدمى وربط المنهح التعليمى بخدمة المحتمع.

\section{Aatl}

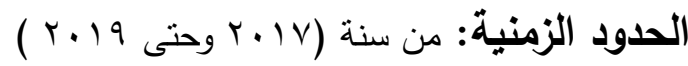

الحدود المكانية: سوف تتطبق هذه الدراسة على عينة من طلبة الفرقة الرابعة بالجامعة

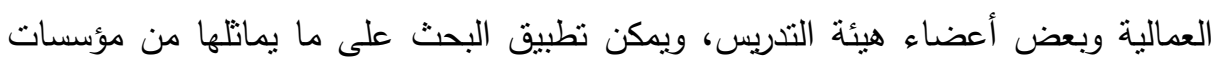

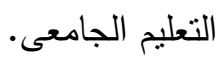

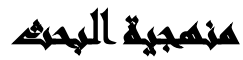

بناء على الهدف الرئيسى للبحث سوف تعتمد على أسلوبين: الأول هو المنهج الوصفى

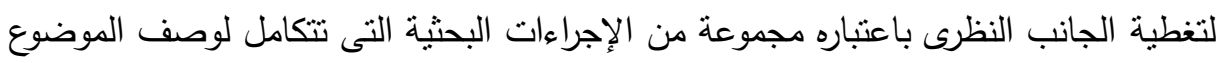

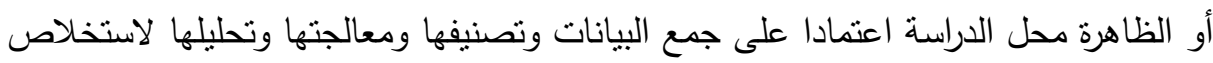
دلالاتها والوصول لنتائج عنها عن طريق، المصادر العربية والأجنبية والكتب والدوريات وشبكة الإنترنت، والأسلوب الثانى يعتمد على منهح البحوث الاستطلاعية من خلال استبانة (مقياس ) ALFA أما فيما ينعلق بالمعالجة الإحصائية فتم معالجة البيانات إحصائيا

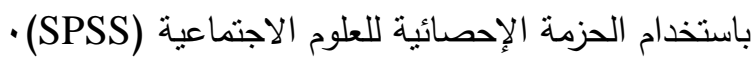




\section{أهمية المهمه}

1- تساعد الدراسة الحالية على تفهم التعلم الخدمى وأهميته مما ينمى اتجاهات القائمين على التعليم نحو تطبيقه.

r- التأكيد على أهية التعلم الخدمى فى تطوير الخدمات التعليمية.

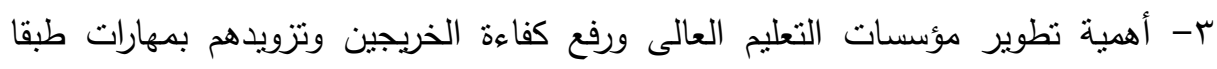

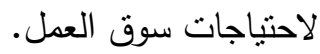

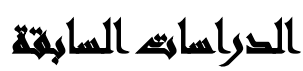

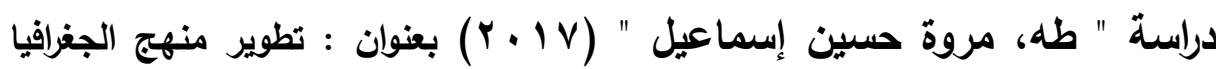
للصف الأول الثانوي في ضوء معايير التعلم الخدمي، نتاول هذه الدراسة تطوير منهج

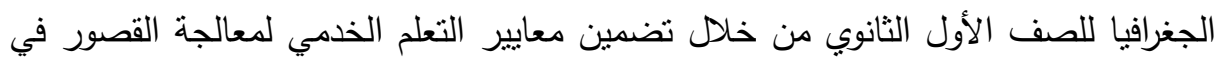

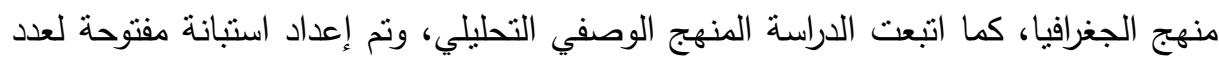

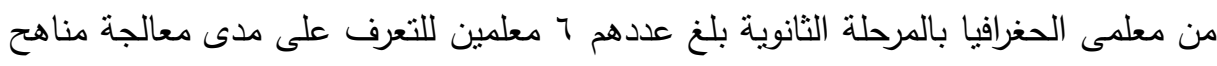
الحغرافيا لمشروعات التعلم الخدى ومدى إدراك هؤلاء المعلمين لأهمية تضمين إستراتيحية التعلم الخدمى فى الفصول الدراسية، وقد أوصى البحث على تضمين معايير التعلم الخدمي

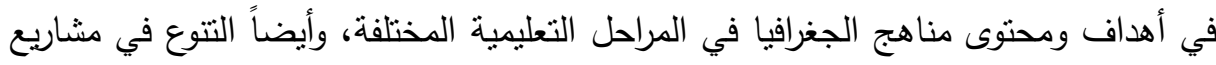
التعلم الخدمي لنتمية المهارات المختلفة التي تلبي احتياجات المجتمع، وكذللك الربط بين منهج الجغرافيا والتدريبات المهنية في التعليم.

دراسة " منصور، سمية حيدر" (7 1 ـ ب) بعنوان متطلبات نجاح التعلم الخدمى كمدخل معاصر فى تربية المواطنة الفعالة فى التعليم : إطار نظرى وتهاف هذه الدراسة الى معرفة

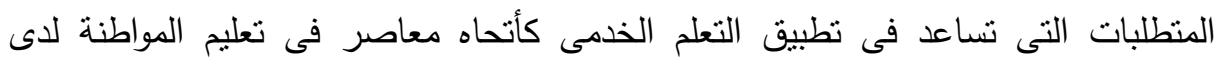
الطلاب وإكسابهم مهاراة التفكير لحل المشكلات والتفاعل مع الاخرين والتعايش معهم لهاه وممارسة دورهم كمواطنين فاعلين فى الحياة المحتمعية وعلى المستوى الثخصى ايضا وهذا

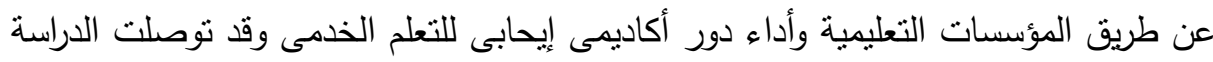
الى عدة نتائح أهمها أن التعلم الخدمى من أبرز المداخل المعاصرة الفعالة فى تربية المواطنة 
وان من متطلبات نحاحه هو نشر نقافته فى المحتمع بشكل عام وعلى المدارس والمؤسسات التعليمية بشكل خاص وكذلك تدريب المعلمين وإعدادهم بطرق تدريسية معاصرة كالتعلم - الخدمى دراسة Terence lovat and Neville Clement (צ 1 • ب ): بعنوان: "لتعلم الخدمى كتربية شاملة للقيم" أوضحت هذه الدراسة قيمة التعلم الخدى كهدف من أهداف التربية وتحقيق تتمية الوعى للمواطنة وربط الطلاب بحاجات المجتمع الذين يعيشون فيه وربط الدراسة الأكاديمية بالمجتمع، وأكدت على أن التعلم الخدمى يعد ركيزة أساسية فى تعلم الطلاب قيم المسئولية والمواطنة وتطوير الذات والثخصية لدى الطلاب، لأن تعلم الخدمة

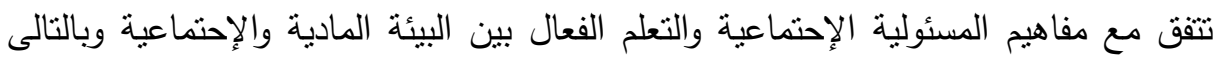
علم الخدمة يساعد على تتمية الثباب وتعليمهم الإنخراط فى المحتمع وهذا يرتبط ارتباطا وثيقا

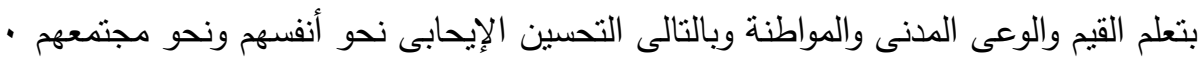
دراسة Wee,Zakaria خبرات التعلم Promoting Civic Engagement Through A Service Learning Experience هافت هذه الدراسة الى التعرف على السلوكيات المدنية للمشاركة المدنية للطلاب فى برامح

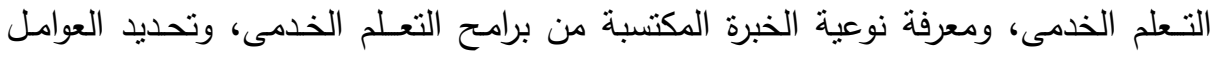

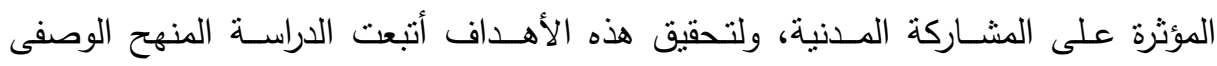

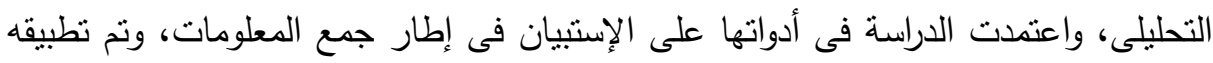

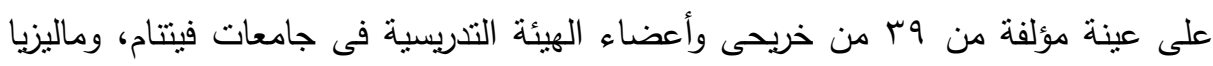
وتايلاند وسنغافورا ممن شاركوا فى برامح التعلم الخدمى وكان من أبرز ما نوصلئ واعلت اليه

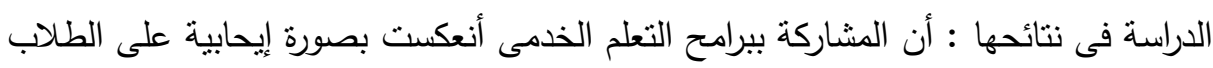

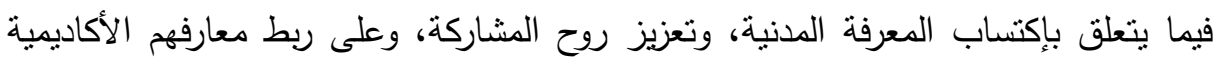

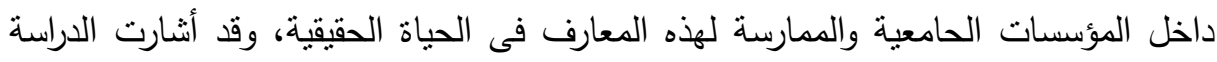


الى ضرورة تـعزيز المشـاركة المدنيـة داخــل المؤسسات التعليمية بصورة مستمرة مدى الحياة

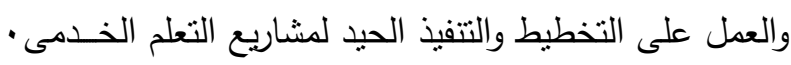

\section{الإسار النظارى}

لما كان هذا البحث يهدف الى تطوير خدمات التعليم العالى فى ضوء مفهوم التعلم

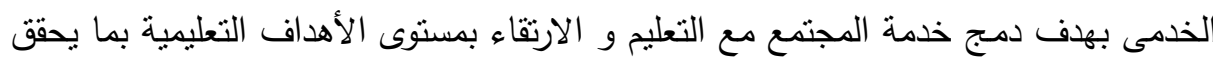
التفاعل والتكامل بين الطالب والمدرس والبيئة المحيطة من خلال المؤسسة التعليمية والتى لهي تهدف الى الوقوف على احتباجات المجتمع ودراسة قضاياه وتلبية مطالب سوق العمل، فلابد أن نتعرف على بعض المفاهيم والتى تساعدنا على معرفة ما نصبوا اليه من تحقيق هذا

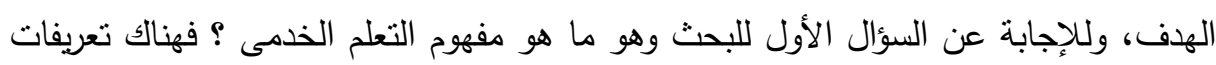

$$
\text { كثيرة للتعلم الخدمى نذكر بعض منها: }
$$

• تعرف جمعية التربية والتدريب والبحث بالولايات المتحدة الأمريكية، التعلم الخدمى بأنه إستراتيجية تعليم وتعلم، تدمج خدمة المجتمع دع التعليم والتامل لإثراء تجربة التعلم وتعلم

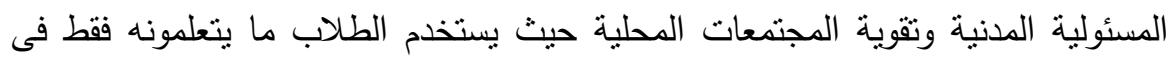
التطبيقات العملية بل يصبحون مواطنبن مساهمين فى بناء المجتمع من خلال الخدمة

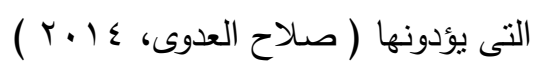

• يعرفه • Mari,Watkins and Linda Braun (2005 بأنه شكل من أنكال التعلم

القائم على الخبرة حيث تحدث عملية التعلم من خلال سلسلة من الأنشطة التى يقوم بها الطلاب مع زملائهم لنطبيق المعرفة التى تعلموها فى حل المشكلات الأجتماعية والتامل

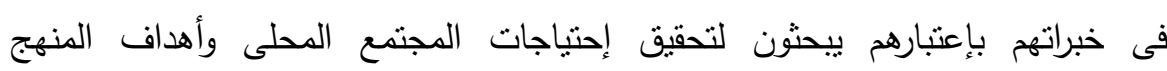
• الدراسى • Hanrahan, بأن التعلم الخدمى يقوم على المنفعة المتبادلة بين

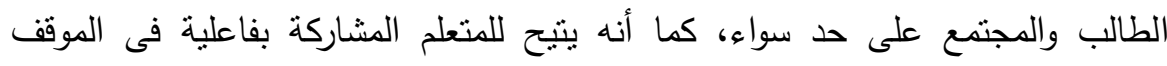

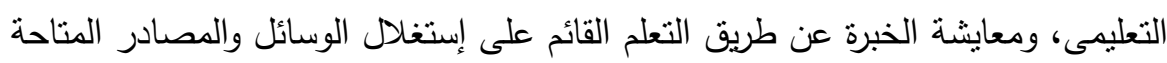
بالمجتمع المحلى · ومعايته 


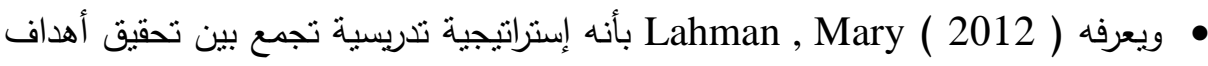

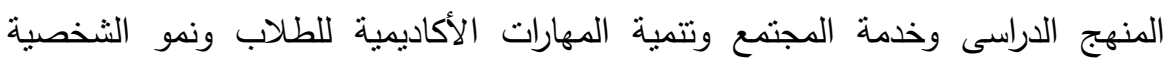
وتحقيق المشاركة المدنية

وظهر التعلم الخدمى كفكرة للتعلم القائم على خدمة المجتمع فى الدول الغربية فى أوائل التسعينات على يد العالم John Dewey, Hilda Taba حيث أعتبر التعلم ما هو إلا نتيجة

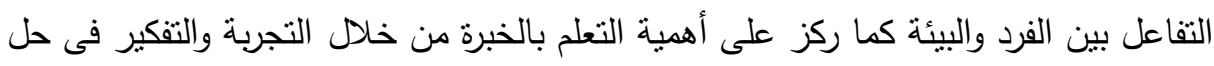

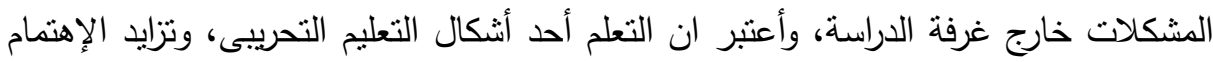

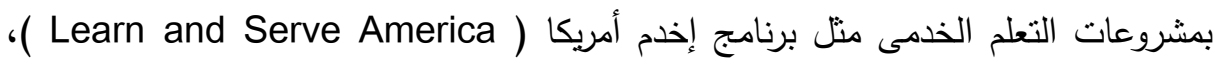
الذى كان يقدم . . . دولار منحة لحوالى مليون طالب من المدارس الثانوية الذين يقومون

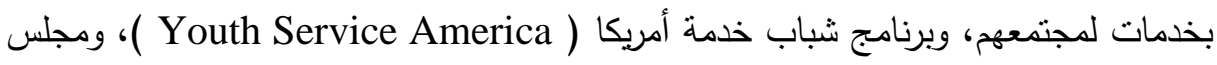
القيادة الوطنى للثباب ( National Youth Leadership Council ) وشبكة التعلم الخدمى وبرنامج إفعل شيئًا ( Do Something )، الذى يسعى أن يكتسب الطلاب المهارات والمواد الضرورية لتحسين مجتمعاتهم وتعزيز العدالة الإجتماعية(العدوى، ـ ـ ـ ب ) ).

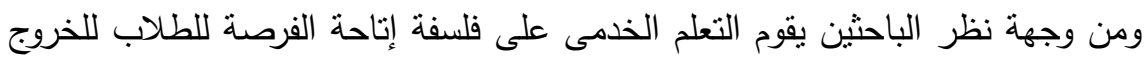
بالعملية التعليمية خارج إطار جدران الجامعة وإستغلال إمكانيات البيئة المحيطة لربط المنهج

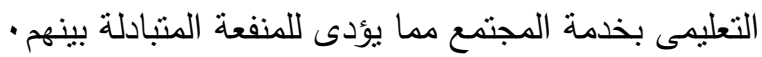

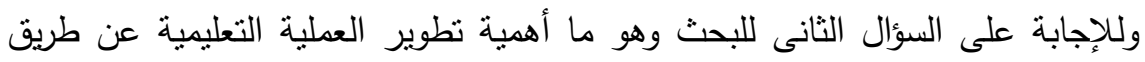
التعلم الخدمى ؟ أكدت معظم الدراسات أن التعلم الخدمى يساعد على تحسين التحصيل التعليمى الأكاديمى لتتمية مهارات التفكير والتامل و تتمية مهارت التعاون والإتصال من خلال التصل العمل الجماعى و غرس روح الإنتماء والمواطنة فى نفوس الطلبة و تتمية المسئولية

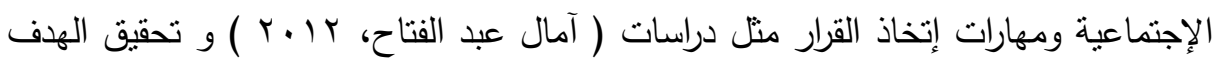
من المنهج الأكاديمى وأهداف المجتمع و تتمية وزيادة الدافع لدى الطلاب لتحمل المسئولية

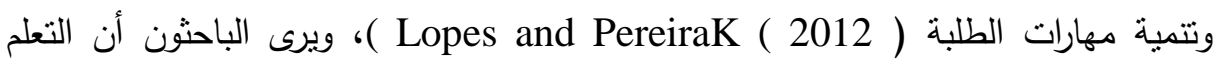
الخدمى يختلف عن الخدمة المجتمية فى أن التعلم الخدمى يرتبط بالمنهج الدراسى مباشرة

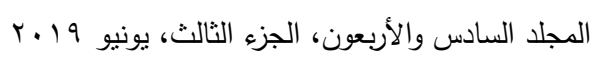


بتكامل وتفاعل بينهم من خلال أهداف واضحة تربط المتعلم بانشطة تخدم المجتمع وإثارة

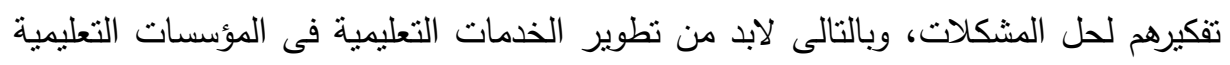

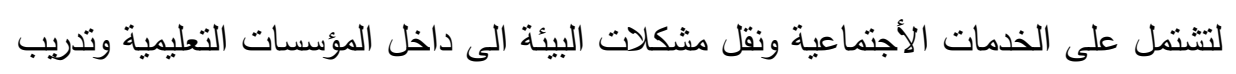
الطلاب على مهارات التواصل.

\section{إجباءاهي المهشه}

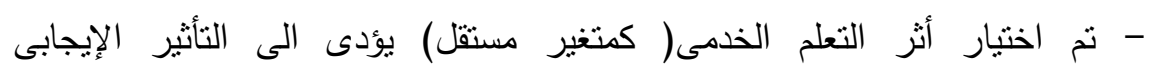
فى(المتغيرات التابعة له ) وهى: أ- تتمية مهارات الطلبة وقدرتهم على التواصل وإكسابهم

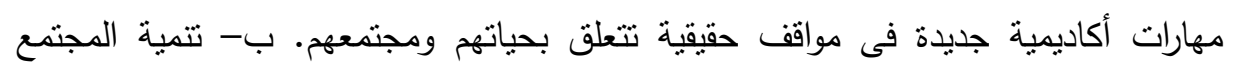
والبيئة المحيطة بمساعدة الطلاب فى مشاريع الخدمة الوطنية. ج- رفع كفاءة أداء القائمين بالتدريس و ربط المنهج التعليمى بمجتمع الطلبة الذين يعيشون فيه وجعلهم يشاركون بتعليمهم

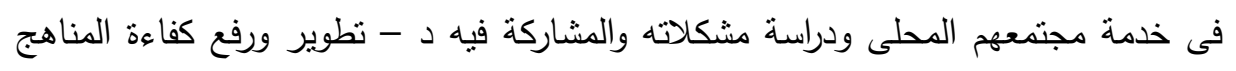

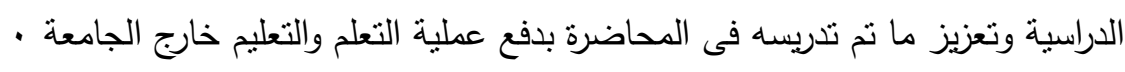

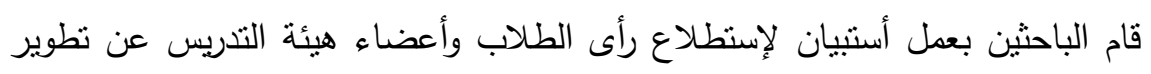

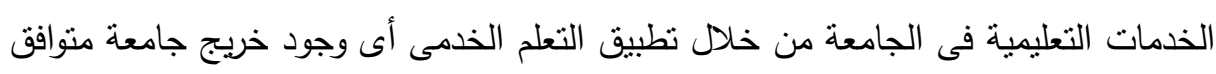
ومتفاعل ومشارك فى حلول مشكلات مجتمعه حيث تعد المشاركة المجتمعية واحدة من فن

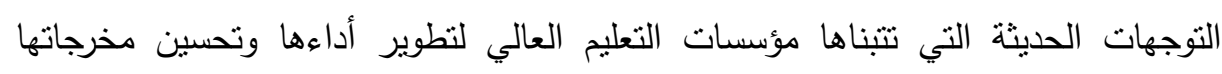

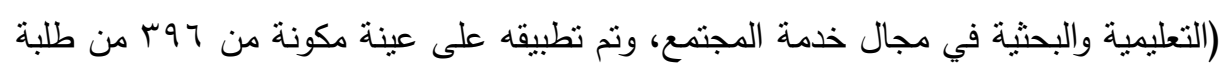

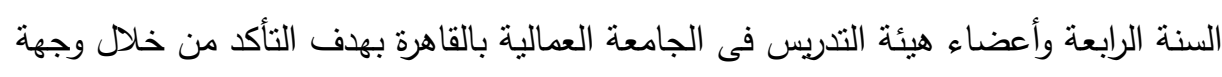

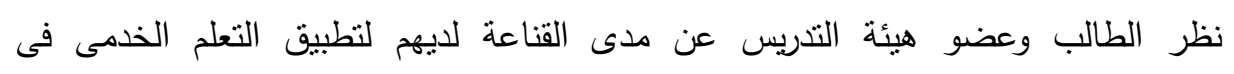

\section{المنيأئم}

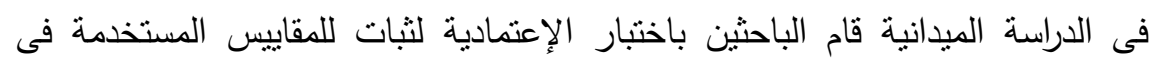

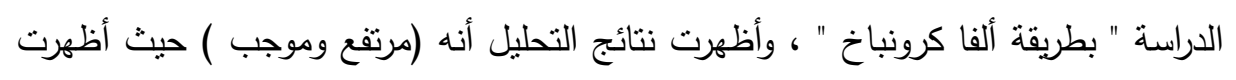


نتائج تحليل الإعتمادية نسبة معامل الثبات لعبارات الأستنيان كلها = r؟ أو وهو مؤشر على

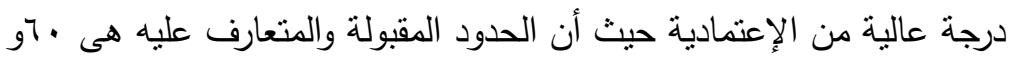
جدول( ) (1): اختبار الإعنمادية الإعنة

\begin{tabular}{|c|c|c|c|}
\hline معامل الفا & عدد العبارات & كود الترميز & المتغيرات \\
\hline .775 & 10 & A & التعلم الخدمى \\
\hline .919 & 10 & $\mathrm{Y} 1$ & تتمية مهارات الطلبة \\
\hline .924 & 10 & $\mathrm{Y} 2$ & رفع كفاءة القائمين بالتدريس \\
\hline .858 & 10 & $\overline{\mathrm{Y} 3}$ & تطوير المناهج \\
\hline .943 & 40 & & المقياس الكلي \\
\hline
\end{tabular}

ثم قاموا الباحثين بعرض " الإحصاءات الوصفية " ( المتوسط الحسابى والانحراف المعيارى ) لجميع متغيرات الدراسة ، وكذلك تم عمل الجداول التكرارية لمجموعة الاسئلة لتحديد عدد مرات التكرار والنسبة المئوية ، وذللك بالتطبيق على آراء المستقصى منهم بطريقة " مقياس ليكارت الخماسى" وبعد دراسة الجداول تنين أن المتغير ( تتمية مهارات الطلبة )

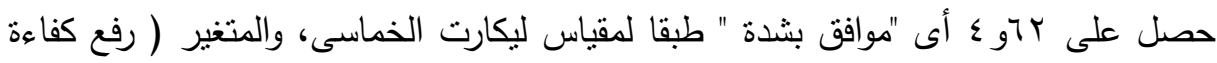
العاملين بالتدريس ) حصل على بآو ع ايضا " موافق بشدة " والمتغير ( تطوير المناهج )

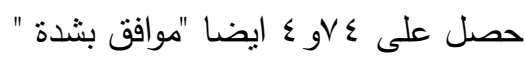
جدول(ץ): لحساب المتوسط الحسابى والانحراف المعيارى لجميع متغيرات الدراسة

\begin{tabular}{|c|c|c|c|c|}
\hline \multicolumn{5}{|c|}{ Descriptive Statistics } \\
\hline آسم المتغير & كود الترميز & $\mathbf{N}$ & Mean & Std. Deviation \\
\hline التعلم الخدمى & A & 396 & 3.43 & .292 \\
\hline تتمية مهارات الطلبة & $\mathrm{Y} 1$ & 396 & 4.62 & .325 \\
\hline رفع كفاءة القائعين بالتذريس & $\overline{\mathrm{Y} 2}$ & 396 & 4.63 & .336 \\
\hline تطوير المناهح & $\mathrm{Y} 3$ & 396 & 4.74 & .252 \\
\hline
\end{tabular}

وقد تبين ايضا من عبارات أسئلة الإستنيان منل عبارة ( أن الكادر العلمى الذى تحصل

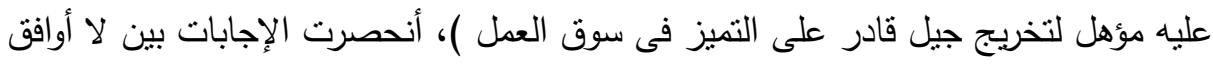

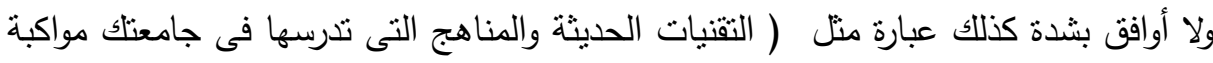
للنطور العلمى ومرتبطة بسوق العمل ) أخذت نفس الإجابات لا أوافق ولا أوافق بثدة وأيضا لـان 
عبارة ( هل تعتقد ان هناك مجالات للعمل الخدمى فى جامعتك ) تتحصر بين لا أوافق ولا أوافق بثدة، وهذا يدل على التالى:

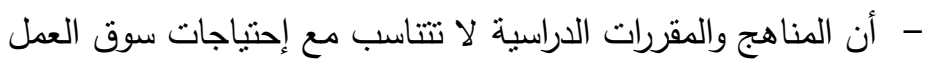

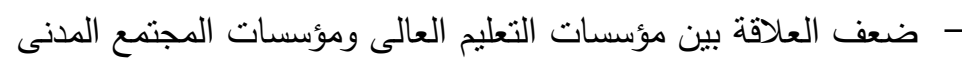

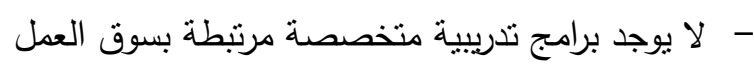

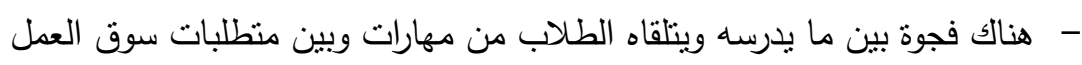

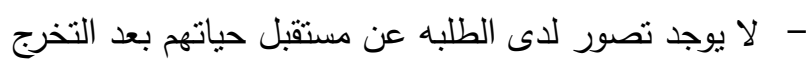

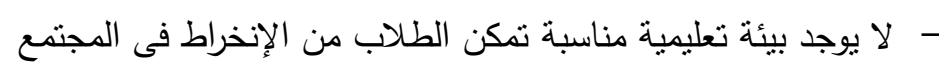
- زيادة التخصصات النظرية عن العملية والتطبيقية - المناهج الدراسية لا نتمى مهارات الطلاب الفكرية وبالتالى يمكن التوصية بتطبيق التعلم الخدمى للأستفاده منه فى نتمية مهارات الطلبه ورفع كفاءة العاملين بالتدريس وربط المنهج التعليمى بخدمة المجتمع · لئل

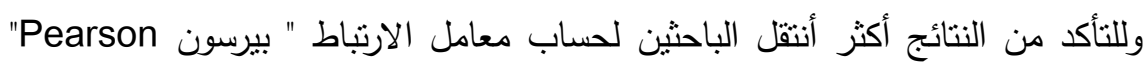

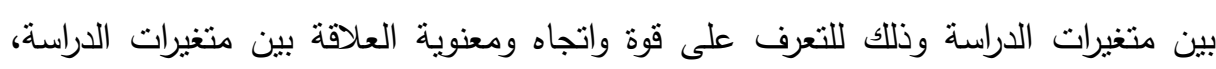

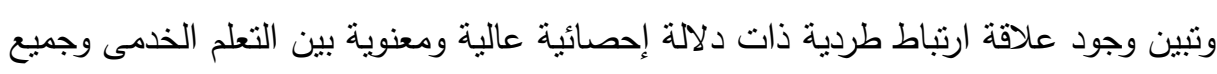

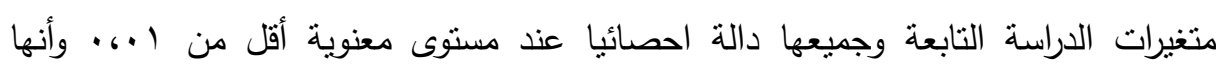

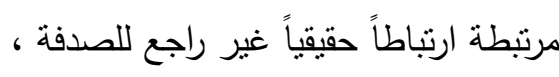

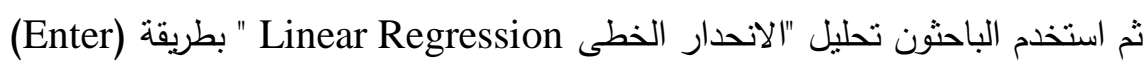

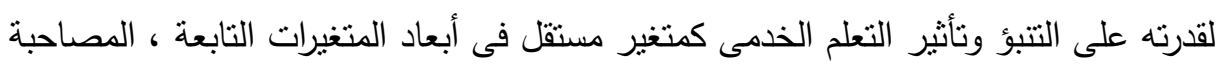

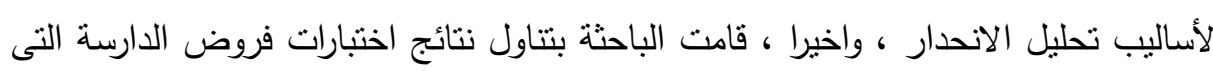
رفضت فرض العدم وقبول الفرض البديل القائل: - يوجد فرق ذو دلالة إحصائية عند مستوى معنوية م = (ه....) بين التعلم الخدمى وتتمية

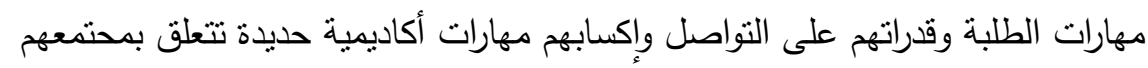
- يوجد فرق ذو دلالة إحصائية عند مستوى معنوية م = (ه....) بين التعلم الخدىى ورفع كفاءة القائمين بالتدريس وربط المنهج التعليمى بخدمة المجتمع دئه 
جearson جدول(ץ): لحساب معامل الارتباط " بيرسون

\begin{tabular}{|c|c|c|c|c|c|}
\hline \multicolumn{6}{|c|}{ Correlations } \\
\hline & & الخدمى التعلم & تنمية مهارات & رالقائمين كفاءة & المناهير \\
\hline \multirow{3}{*}{ التعلم الخدمى } & $\begin{array}{c}\text { Pearson } \\
\text { Correlation } \\
\end{array}$ & 1 & $.362 * *$ & $.363 * *$ & $.285 * *$ \\
\hline & Sig. (2-tailed) & & .000 & .000 & .000 \\
\hline & $\mathrm{N}$ & 396 & 396 & 396 & 396 \\
\hline \multirow{3}{*}{ تتمية مهارات } & $\begin{array}{c}\text { Pearson } \\
\text { Correlation }\end{array}$ & $.362 * *$ & 1 & $.818 * *$ & $.629 * *$ \\
\hline & Sig. (2-tailed) & .000 & & .000 & .000 \\
\hline & $\mathrm{N}$ & 396 & 396 & 396 & 396 \\
\hline \multirow{3}{*}{ في كفاءة القائمين } & $\begin{array}{c}\text { Pearson } \\
\text { Correlation }\end{array}$ & $.363 * *$ & $.818 * *$ & 1 & .754 \\
\hline & Sig. (2-tailed) & .000 & .000 & & .000 \\
\hline & $\mathrm{N}$ & 396 & 396 & 396 & 396 \\
\hline \multirow{3}{*}{ تطوير المناهح } & $\begin{array}{l}\text { Pearson } \\
\text { Correlation }\end{array}$ & $.285^{* *}$ & $.629 * *$ & $.754 * *$ & 1 \\
\hline & Sig. (2-tailed) & .000 & .000 & .000 & \\
\hline & $\bar{N}$ & 396 & 396 & 396 & 396 \\
\hline
\end{tabular}

ويتضح من الجدول السابق، وجود علاقة ارتباط طردية ذات دلالة إحصائية عالية ومعنوية بين التعلم الخدمى وتتمية مهارات الطلبة وكذلك وجود علاقة طردية معنوية بين التعلم الخدمى ورفع كفاءة العاملين بالتدريس وربط المنهح التعليمى بخدمة المحتمع وجميعها دالة

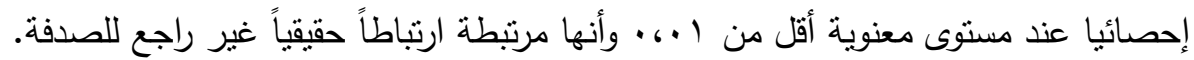

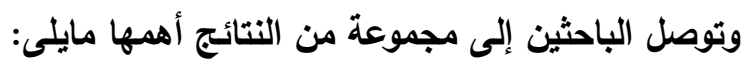
1- التعلم الخدى يعتبر مدخلا لتطويرالخدات التعليمية ومناهج التعليم الجمى فى الجامعات المصرية ويحقق الأهداف الأكاديمية للتعلم بصورة فعالة

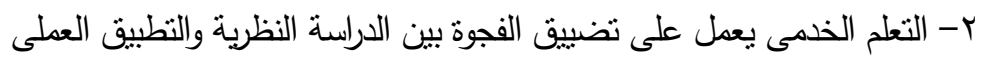

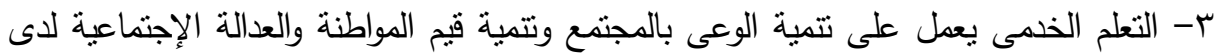
الطلاب 
ع- يساعد التعلم الخدى فى إتاحة الفرصة للطلاب للتأمل الذاتى فى المواقف الحيانية المختلفة حيث

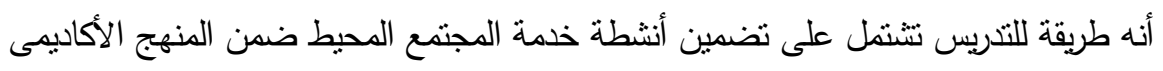

$$
\text { الذى يدرسونه }
$$

0- يساهم التعلم الخدمى فى نوفير بيئة محفزة ومشجعة للتعلم צ- يمكن التعلم الخدى الطلاب من التعرف على المهن المستقلية المتاحة فى المجتمع مما يساعد

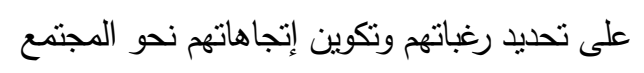

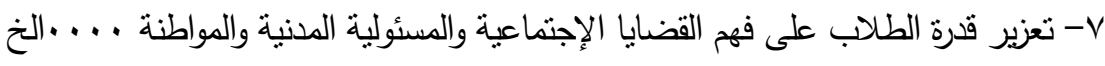

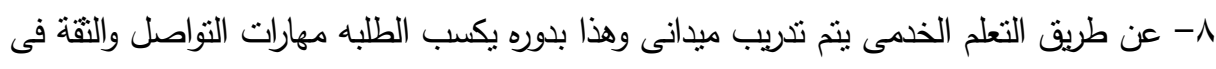

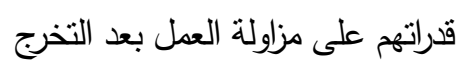

9- التعلم الخدى يحقق التوازن بين أهداف التعليم والتعلم واهداف الخدمة فى نفس الوقت فهو تعلم

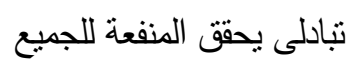

• 1- تعلم يقوم على منهج أكاديمى بجانب خدمة مجتمعية وبالتالى فليس كل عمل اولى خدمة مجتمعية تصلح نكون تعلم خدمى لأن التعلم الخدى مرنبط بمنهج أكاديمى تعليمى تعاونى بين

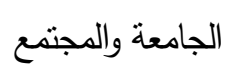

1) - أظهرت الدراسة أن هنالك وجود علاقة طردية ذات دلالة إحصائية بين التعلم الخدى وبين كل

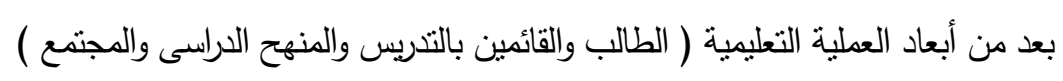

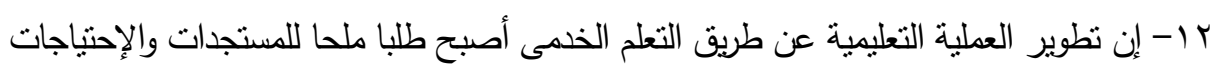
المجتمعية وتخريج جيل فادر على هذه المنطلبات ولا يكون عبئا على المجتمع حتى تتمكن

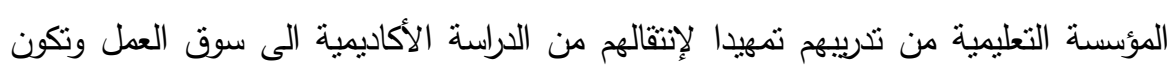

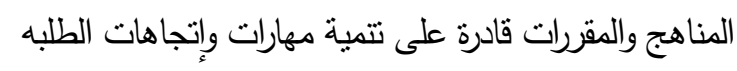




\section{التهوصياهت}

فى ضوء نتائج الدراسة السابقة قاموا الباحثين بتقديم مجموعة من التوصيات طبقا لما

$$
\text { توصلت اليه والتي يمكن توضيحها فى التالي: }
$$

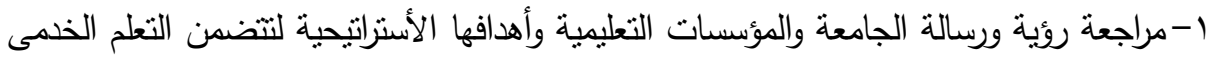

وتوفير الإمكانيات والتسهيلات التعليمية اللآزمة لذلك، بحيث يكون هنالك نموذج نعليمى موحد

$$
\text { ب-ربط الأبحاث الى تضمين التعلم الخدمى ضمن مقررات التعليم الأكاديمى العلى }
$$

r-ضرورة التدريب الميدانى لإكساب الطلبة مهارات التواصل والثقة بالنفس والقدرة على مزاولة المهن

$$
\text { والعمل بعد التخرج }
$$

ع - إنثاء جامعات جديدة ببرامح غير تقليبية لنليية سوق العمل

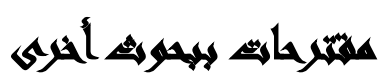

إعداد دراسة بحثية لوضع نموذح لتطوير اللائح الدراسية بالحامعات المصرية بما

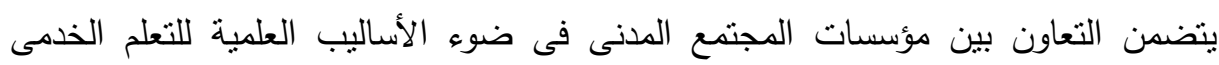

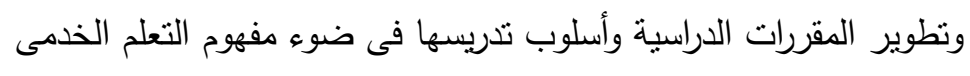

\section{zall}

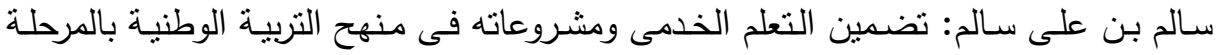

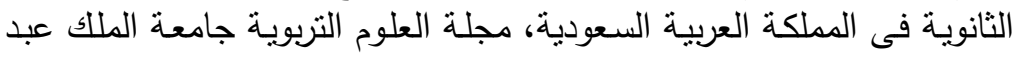

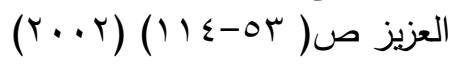

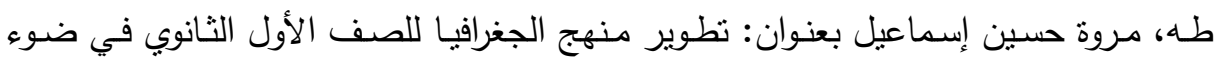

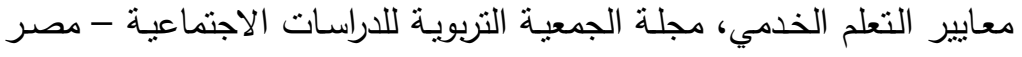

$$
(r \cdot 1 v)(v 0-r q)
$$

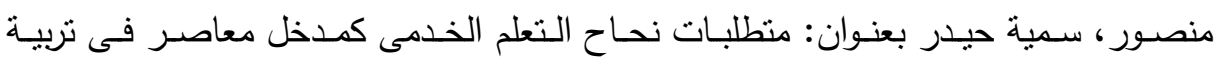

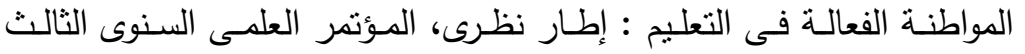

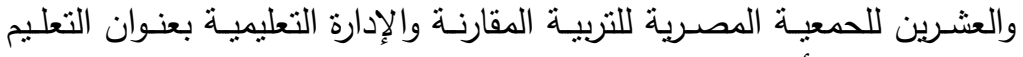

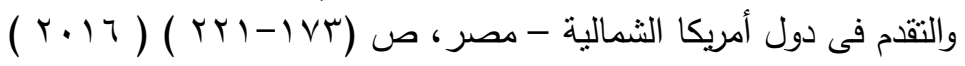

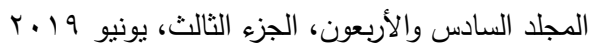




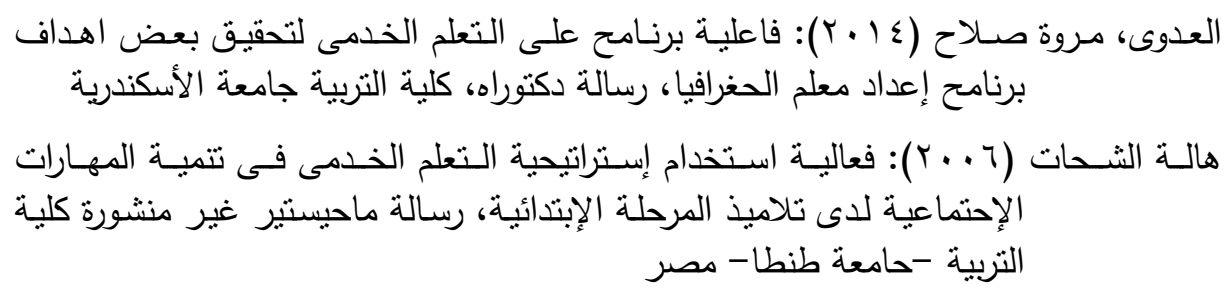

Lovat, Terence; Clement, NevilleService Learning as Holistic Values PedagogyJournal of Experiential Education, v39 n2 p115129 Jun 2016. 15 pp, (2016).

Wee,Zakaria Promoting Civic Engagement Through A Service Learning Experience (2012).

\title{
DEVELOPING HIGH EDUCATION SERVICES IN EGYPTIAN UNIVERSITIES ACCORDING TO SERVICE LEARNING CONCEPT
}

Wafaa F. Abd El-Wahab ${ }^{(1)}$; Nader Albair ${ }^{(2)}$;
Gaber Al-Sharawy ${ }^{(3)}$ and Aymn M. Rashad

1) Workers' University 2) Faculty of Commerce Ain Shams University

3) Faculty of Science, Al Azhar university

\begin{abstract}
Despite the critical significance of education all over the Arab world in general and despite the huge sums of money spent by governments to raise and elevate citizens' position through different various levels of education's programs, Lots of criticisms are referring to low quality and level of educational output in these states. The output of education doesn't cope with development plans, labor market, and high cost of education in light of the scientific low level in educational institutions. University service for society is the actual translation of the university jobs .
\end{abstract}


This present research drives at developing high Education Services In Egyptian Universities According to Service Learning concept its target's is to merge the society service with education and elevating the level of educational objectives which would achieve interaction and integration between student, teacher, and the environment through educational institution which purpose is to stand on society's needs, examining its issues, and fulfilling the labor market's demands.

In order to achieve the objectives of the study, the analytical descriptive approach was followed. The researchers studied the theory and described the problem and analyzed its dimensions and causes using the inductive method and the logical conclusion by collecting data, information and statistics related to the subject of the study through books and references, scientific conferences, published researches, , And draw conclusions. he study showed that there is a statistically significant relationship between service learning and every dimension of the educational process teaching and teaching community) The study recommended the inclusion of a unified educational model for service learning within academic curricula, linking scientific research to community service and the need to train graduates with non-traditional labor market programs. 